This document is published in:

Economics Letters (2001), 72(3), 303-307.

DOI: 10.1016/S0165-1765(01)00445-1

(C) 2001 Elsevier Science B.V. 


\title{
Extending communication-proof equilibrium to infinit games
}

\author{
José Luis Ferreira* \\ Department of Economics, Universidad Carlos III de Madrid, 28903 Getafe, Madrid, Spain
}

\begin{abstract}
The concept of Communication-proof equilibrium is extended to infinite games. To that end we make use of abstract stable sets as defined by Greenberg in his Theory of Social Situations.
\end{abstract}

Keywords: Communication-proof; Infinit games; Stable sets

JEL classificatio : $\mathrm{C} 70 ; \mathrm{C} 72$

\section{Introduction}

The concept of Communication-proof equilibrium, Com-PE, was proposed in Ferreira (1996) for finit games. Here we present its extension to infinit games using the approach in Greenberg (1989). To understand this definitio think of a group of people that arrives at an agreement and then must leave the room. The problem is that, when a player leaves, he may rightly suspect that the remaining players will change their actions taking as given his own strategy. A coalition-proof Nash equilibrium, Coalition-PNE, (Bernheim et al., 1987, from now on B,P\&W) is an arrangement that will not be changed regardless of the order of exit. To modify the definitio for extensive form games one needs to take into consideration not only the usual problems of time consistency but the renegotiation issues as well. In particular, if part of the deviation can be observed, the possibility of a reaction by the opponents cannot be ruled out. Unlike the definitio of perfectly coalition-proof Nash equilibrium, $P C o a l i t i o n-P N E$, provided by $\mathrm{B}, \mathrm{P} \& \mathrm{~W}$, the concept of Com-PE reflect these points and can be regarded as an extension of both Coalition-PNE and renegotiation-proof equilibria.

*Corresponding author. Tel.: +34-91-624-9580; fax: +34-91-624-9575.

E-mail address: jlferr@eco.uc3m.es (J.L. Ferreira). 


\section{Notation and definition}

Let $\Gamma$ be an extensive form game with no chance moves with $N=\{1, \ldots\}$ as the set of players. A subset of $N$ will denote a coalition. The (finit ) set of pure strategies for player $i$ will be denoted by $S_{i}$. Strategy profile for sets of players are denoted by $S=X_{i \in N} S_{i}, S_{C}=X_{i \in C} S_{i}$ and $S_{-C}=X_{i \in-C} S_{i}$, where $C \subset N$ is a coalition and $-C=N \mid C$. Their respective typical elements will be $s_{i}, s, s_{C}$ and $s_{-C}$. The vector $\left(s_{-i}, s_{i}^{\prime}\right)$ denotes the strategy profil $s$ in which $s_{i}$ has been replaced with $s_{i}^{\prime}$. Let $u_{i}: S \rightarrow R$ be the outcome function for player $i$ (it will be identifie with his von Neumann-Morgenstern expected utility function). The notation $s>{ }_{C} s^{\prime}$ means that $u_{i}(s)>u_{i}\left(s^{\prime}\right)$ for every player $i$ in the coalition $C$. The game that $s_{C}$ induces on $\Gamma$ (i.e. the game that coalition $C$ faces when $s_{C}$ is regarded as fixe ) will be denoted by $\Gamma / s_{C}$. A subgame of $\Gamma$ is indicated by $g$.

The number of stages in the game is the maximum number of nested subgames in it. Therefore a stage is the play between two consecutive nested subgames. By $s(t)$ we will denote the behavior that strategy $s$ induces at stage $t$.

The set of histories when the game starts (stage 1 ) is given by $H_{1}=\{0\}$. The set of feasible histories $H$ for a $\tau$ stage game is given by $H=\cup_{t=1}^{\tau} H_{t}$; where $H_{t}$ is define recursively by

$$
H_{t+1}=\left\{h_{t+1} \quad \mid \quad \exists h_{t} \in H_{t} \text { s.t. } h_{t+1} \in\left\{h_{t}\right\} \times S^{h_{t}}\right\},
$$

where $S^{h_{t}}=X_{i=1}^{n} S_{i}^{h_{t}}$ and where $S_{i}^{h_{t}}$ is the set of actions for player $i$ in stage $t$ given $h_{t}$. Some more notation about histories include: The set of feasible histories following $h, H^{h}$; the subgame induced by history $h, g^{h}$; the strategy induced by $s$ after history $h, s^{h}$; the stage at which history $h$ is observed, $t(h)$ (history $h$ ends at stage $t(h)-1$ ) and the set of histories of length $t(h)+1$ that belong to $H^{h}, h_{1}$.

When there is no confusion, the short name of an equilibrium (e.g. $S P E$, after subgame perfect equilibrium) will denote the set of those equilibria in a given game.

In what follows, games will be assumed to be of complete information and perfect recall so that only behavioral strategies will be considered.

Definitio 1. (Extension of Coalition-PNE in B,P\&W) (i) In a single player game, $s^{*}$ is a Coalition-proof Nash equilibrium restricted to $R \subset S$, Coalition-PNE(T), if $s^{*} \in \operatorname{argmax}_{s \in R} u^{1}(s)$.

(ii) Assume that Coalition- $P N E(R)$ has been define for games with less than $n$ players. Then, in a $n$-players game;

(a) $s * \in S$ is Self-enforcing restricted to $R, S E(R)$, if for any coalition $C \neq N, s$ is Coalition$P N E(R)$ in the game $\Gamma / s_{-C}^{*}$;

(b) $s * \in S$ is Coalition-PNE(R) if it is $S E(R)$ and if it does not exist in any other $s \in R$ such that $s$ is $S E(R)$ and $u^{i}(s)>u^{i}\left(s^{*}\right)$ for all $i \in N$.

Definitio 2. (i) In a single stage game $\Gamma, s *$ is a Communication-proof equilibrium, Com- $P E$, if it is a Coalition-PNE.

(ii) Let $t>1$ and assume that Com-PE has been define for games with $r<t$ stages. Then, in a game $\Gamma$ with $t$ stages, $s^{*}$ is Com-PE if it is a Coalition-PNE $\left(S^{1}\right)$ where $S^{1}=\{s \in S \mid s$ induces a Com- $P E$ in proper subgames of $\Gamma$ \}. 


\section{Extending Com-PE to infinit games}

This section follows the approach by Greenberg (1989) where the stable sets are used to extend recursive definition to the infinit case.

A von Neumann and Morgenstern abstract system $(A S)$ is a pair $(D,>)$ where $D$ is an abstract set and $>$ is a dominance relation $(f>d$ will be read as ' $f$ dominates $d$ '). Let $(D,>)$ be an abstract system, and let $f \in D$. The dominion of $f$, denoted by $\Delta(f)$, is the set $\Delta(f)=\{d \in D \mid f>d\}$. That is, $\Delta(f)$ consists of all elements of $D$ that $f$ dominates, according to the dominance relation $>$. Similarly, for a subset $F \subset D$, the dominion of $F$, denoted by $\Delta(F)$, is the set $\Delta(F)=\cup$ $\{\Delta(f) \mid f \in F\}$. That is, an element $d$ in $D$ belongs to $\Delta(F)$ if it is dominated by some element in $F$. A set $F \subset D$ is a von Neumann and Morgenstern abstract stable set $(A S S)$ for the system $(D,>)$ if $F=D \mid \Delta(F)$.

Let $\Gamma$ be a multi-stage game. Inspired by Definitio 2 an abstract system $(D,>)$ is introduced. Let the elements of the abstract set consist of a coalition, a subgame and a strategy profil in this subgame,

$$
D=\left\{\left(C, g^{h}, s\right) \quad \mid \quad C \subset N, C \neq \emptyset, h \in H, s \in S^{h}\right\}
$$

where $S^{h}$ is the set of strategy profile in the subgame after $h$; and let the domination relation be define as follows: $\left(B, g^{k}, y\right)>\left(C, g^{h}, s\right)$ if and only if either

1. $k \in H^{h}$ and $k \neq h: B \subset N, s_{-B}=y_{-B}, y^{k}>_{B} s^{k}$ or

2. $k=h: B \subset C, s_{-B}=y_{-B}, y^{k}>_{B} s^{k}$.

The next proposition (the proof of which is just the proof of the lemmas after it) relates the $A S S$ of $(D$, $>$ ) with the definitio of Com-PE for finit games and allows for a definitio applicable to infinit games.

Proposition 1. Let $K$ be an ASS of $(D,>)$; then, for finit games, we have that for all $h \in H, C \subset N$ and $s \in S ;\left(C, g^{h}, s\right) \in K$ if and only if $\left(s_{C}(t(h)), s^{h_{1}}\right)$ is a Com-PE in the subgame $g^{h} / s_{-C}(t(h))$. In particular, when $C=N$ and $h=\emptyset, \operatorname{Com}-P E(\Gamma)=\{s \quad \mid \quad(N, \Gamma, s) \in K\}$.

Lemma 1. For all $h \in H, C \subset N$ and $s \in S$; if $\left(s_{C}(t(h)), s^{h_{1}}\right)$ is a Com-PE in $g^{h} / s_{-C}(t(h))$ then there are no $B, k$ and $x$ such that $\left(x_{B}(t(k)), x^{k_{1}}\right)$ is a Com-PE in $g^{k} / x_{-B}(t(k))$ and neither of the following is satisfied

(i) $k \in H^{h}, B \subset N, s_{-B}=x_{-B}, x^{k}>{ }_{B} s^{k}$

(ii) $k=h, T \subset C, s_{-T}=x_{-T}, x^{k}>{ }_{T} s^{k}$

Proof. The proof is by induction in the number of stages $t$. If $t=1$, the proof reduces to show that for all $C \subset N, s_{C}$ is a Com-PE (=Coalition-PNE) in $\Gamma / s_{-C}$ implies that no $B \subset N$ and $x \in S$ exist such that $x_{B}$ is Coalition-PNE in $\Gamma / x_{-B}$ and $B \subset C, x_{-B}=s_{-B}, x>_{B} s$; but this comes from Lemma 1 in Greenberg (1989).

Assume that the Lemma has been proved for games with strictly less than $t$ stages and prove it now 
for $t>1$. If $\left(s_{C}(t(h)), s^{h_{1}}\right)$ is a Com-PE in the restricted game $g^{h} / s_{-C}(t(h))$ and $k \in H^{h}$ and if $k \neq h$ then $s^{k}$ is a Com-PE in $g^{k}$ by definitio of Com-PE. Apply the induction hypothesis to get that no $B \subset N$ and $x \in S$ exist such that $\left(x_{B}(t(k)), x^{k_{1}}\right)$ is a Com-PE in $g^{h} / x_{-B}(t(k))$ with $x_{-B}=s_{-B}$ and $x^{k}>{ }_{B} s^{k}$. Finally, for the case $k=h$, suppose that there exist a coalition $B \subset N$ and a strategy profil $x \in S$ such that $\left(x_{B}(t(h)), x^{h_{1}}\right)$ is a Com-PE in $g^{h} / x_{-B}(t(h))$, then $x^{1}$ (the restriction of $x$ in $g^{h_{1}}$ ) is a Com-PE in $g^{h_{1}}$; if $B \subset C, s_{-B}=x_{-B}$ and $x^{k}>_{B} s^{k}$ then $s$ was not a Coalition-PNE restricted to $S^{\prime}=\{s \in S \quad \mid \quad s$ induces a Com-PE induces a Com-PE in proper subgames of $\Gamma\}$.

Lemma 2. For all $h \in H, C \subset N$ and $s \in S$, if $\left(s_{C}(t(h)), s^{h_{1}}\right)$ is not a Com-PE then there exist $B \subset N$, $k \subset H$ and $x \in S$ such that $\left(x_{B}(t(k)), x^{k_{1}}\right)$ is a Com-PE in $g^{h} / s_{-B}(t(k))$ and either

1. (i) $k \in H^{h}, k \neq h, s_{-B}=x_{-B}$ and $x^{k}>_{B} s^{k}$ or

2. (ii) $k=h, B \subset C, s_{-B}=x_{-B}$ and $x^{k}>{ }_{B} s^{k}$.

Proof. If $t=1$, it comes from Lemma 2 in Greenberg (1989).

If $t>1$, that $\left(s_{C}(t(h)), s^{h_{1}}\right)$ is not a Com-PE in $g^{h} / s_{-C}(t(h))$ means that either

(i') the restriction of $s$ in $g^{k}\left(s^{1}\right)$ is not a Com-PE in $g^{k}$ with $k \neq h, k \in H^{h}$, or

(ii') $s^{1}$ is a Com-PE in $g^{h_{1}}$ but there exist $B \subset C$ and $x_{B} \in S_{B}$ such that $\left(x_{B}, s_{-B}\right)^{k}>{ }_{B} s^{k}$ and $\left(x_{B}\right.$, $s_{-B}$ ) is a Com-PE in $g^{h} / s_{-B}(t(h))$.

(i') implies (i) by induction hypothesis and (ii') implies (ii) by setting $x=\left(x_{B}, s_{-B}\right)$.

Since this characterization of a Com-PE is not based on backward recursion, it can be used to formulate a general definition covering both finit and infinit games (in the number of stages and players).

Definitio 3. Consider a multi-stage game $\Gamma$ with a finit or infinit number of players. A strategy profil $s$ is said to be a Com-PE of $\Gamma$ if and only if there is an $A S S, F$, for the associated system $(D$, $>$ ) such that $(N, \Gamma, s) \in F$.

Proposition 2. Let $L$ and $M$ be abstract stable sets of the abstract set $(D,>)$ associated with a finit horizon game, as define above, then $L=M$.

Proof. Let $\left\{\left(C_{i}, g^{h_{i}}, s_{i}\right)\right\}_{i}$ be an infinit sequence with $\left(C_{i+1}, g^{h_{i+1}}, s_{i+1}\right)>\left(C_{i}, g^{h_{i}}, s_{i}\right)$ for all $i$. By finitenes of the sets of coalitions and histories and because the domination relation requires that either $C_{i+1} \subset C_{i}$ or $h_{i+1} \in H^{h_{i}}$, we have that, in the tail of the sequence, $C_{i+1}=C_{i}$ and $h_{i+1}=h_{i}$. This tail is clearly transitive. According to corollary $4 \mathrm{~b}$ in Arce and Kahn (1991), this is a sufficien condition for the uniqueness of the abstract stable set if it exists.

Proposition 2 cannot be generalized to infinit horizon games. Perhaps the simplest example to show this is the following. Consider a game consisting of a set of $\{1,2, \ldots\}$ lions, in this order. Each lion can eat the lion before it (except lion 1, that can eat an antelope) or not. If he does not, the game ends, but if he ate the lion before him (or the antelope), the next lion plays. Each lion prefers firs to eat the antelope (directly or by eating the lion immediately before him if he ate the lion that ate the lion ... that ate the antelope) and not to be eaten, second to do nothing and third to be eaten (there is no use in eating a lion that did not eat the antelope directly or indirectly). If there are $n$ lions, there is 
a SPE that depends on whether $n$ is even or odd. If $n$ is even, odd numbered lions decide not to eat and even numbered ones eat the previous lion only if he ate the lion that ate the lion ... that ate the antelope. If $n$ is odd, it is the odd numbered who eat and the even numbered do not eat. If the number of lions is infinite the two equilibria just described are two $S P E$ (and Com-PE) belonging to different abstract stable sets.

Finally, we compare Definitio 3 with that of PCoalition-PNE. For that defin a new abstract system $(D,>*)$ with $D$ as before and with the domination relation $>*$ define by $\left(B, g^{k}, y\right)>*(C$, $\left.g^{h}, s\right)$ if and only if

(3) $k \in H^{h}, B \subset C, s_{-B}=y_{-B}$ and $y^{k}>{ }_{B} s^{k}$.

Definitio 4. (Asheim, 1991) Let $\Gamma$ be a multi-stage game. A strategy $s \in S$ is said to be a PCoalition-PNE of $\Gamma$ if there exists an abstract stable set, $F$, for the associated system $\left(D,>^{*}\right)$ such that $(N, \Gamma, s) \in F$.

The differences between Com-PE and PCoalition-PNE are now clearer. Condition (3) is equivalent to condition (2) when restricted to the stage where the deviation takes place. After the deviation is known $(k \neq h), 3$ is replaced with (1), the condition that allows for reactions against the deviation by any subset of players.

\section{Acknowledgements}

Financial support from DGESIC grant PB-98/0024 (Ministerio de Educación y Cultura) is gratefully acknowledged.

\section{References}

Ferreira, J.L., 1996. A communication-proof equilibrium concept. Journal of Economic Theory 68, $249-257$.

Asheim, B., 1991. Renegotiation-proofness in finit and infinit stage games through the theory of social situations. Discussion Paper A-173, University of Bonn.

Arce, D., Kahn, C., Kahn, D., 1991. The mathematics of semi-stability. Mimeo, University of Illinois at Urbana-Champain. Bernheim, B.D., Peleg, B., Whinston, M.D., 1987. Coalition-proof Nash equilibria I: concepts. Journal of Economic Theory $42,1-12$.

Greenberg, J., 1989. Deriving strong and coalition-proof Nash equilibria from an abstract system. Journal of Economic Theory 49, 195-202. 